\title{
TINTING STRENGTH OF PIGMENTS
}

\author{
By H. D. Bruce
}

ABSTRACT

In this paper there is presented a discussion of the tinting strength of pigments. It is not primarily proposed herein to offer a new method for estimating and expressing tinting strength, but rather to aid in clarifying ideas on the subject, so that when the science of colorimetrics has been further advanced a rational means for appraising this valuable property may be the more easily developed.

The necessity for considering the tinting strength of chromatic pigments as a two-factor property is shown. A distinction between the tinting strength of whites and of blacks is drawn. New names for three differentiated factors involved in tinting are offered, namely, chromatic, darkening, and brightening strengths. A method for treating these properties photometrically or spectrophotometrically and of indicating their relative magnitude by numerical indices is suggested. Tabular and graphical data are presented.

In connection with and incidental to the subject of tinting, certain empirical formulas have been developed in the argument which may be used for computing the proper mixture of white pigment with pigment of another color in order to obtain a tint of some desired brightness or colorimetric purity.

The procedure employed in this work for specifying color in the monochromatic terms of dominant wave length, purity, and brightness is set forth with concise explanation in an appendix.

\section{CONTENTS}

I. Introduction

II. Discussion of tinting strength $\ldots$

III. Experimental data _. _

IV. Discussion of data _... 140

1. Pigments other than white

2. White pigments

V. Appendix

\section{INTRODUCTION}

The color of a paint is the result of a very complex relationship between the optical properties of the pigment particles, the color and refractive index of the vehicle, the ratio of pigment to vehicle, the conditions of observation and illumination, and often the surface covered. Upon the optical properties of the particles depends the pigment's power to diffuse, transmit, selectively absorb, and disperse incident light. These optical properties are determined not only by 
the chemical nature and refractive index, but in a large measure by the size, shape, and texture of the individual grains.

In examining pigments for their value in decorative paints and enamels, where color and tinting strength are primary considerations, chemical composition may be of quite minor importance as compared with physical properties. For example, two chrome yellows, both C. P. lead chromate, may differ so considerably in physical structure that under otherwise identical conditions they will form tints quite unlike in appearance and depth of shade. (Throughout this discussion, the term "depth of shade" or "depth of tint" will be used without rigorous definition to designate the common conception of degree of tint upon which tinting or color strength depends. The terms "tinting strength" and "color strength" are used synonymously in the literature.) It naturally follows that the strength of pigments should be evaluated by physical means, with no great reliance placed upon the results of chemical analysis.

At present the following procedure for determining the tinting strength of pigments is usually employed. A small quantity of the sample to be tested is accurately weighed, transferred to a large glass slab, and mixed by spatula with a measured volume of bleached linseed oil. A weighed amount of zinc oxide is then added and the mixture rubbed with a flat-bottomed muller in an arbitrarily specified manner. An equal weight of a selected standard is treated in the same way. Dabs of each tint are then compared side by side on a glass slide. From the relative depths of shade of the two tints the relative color strengths of the two samples are judged.

There are several objections to this method of testing tinting strength. The method does not readily lend itself to precise quantitative evaluation. To overcome this objection, it has been suggested that the standard should be assigned a value in tinting strength of 1.0, and that the unknown be varied in amount to match exactly the depth of shade produced by the standard. The ratio of the amount of unknown used to the amount of standard would then be taken as the numerical value of the strength of the unknown sample of pigment. There is also Ostwald's suggestion that the strength of color can be measured by the ratio in which chromatic and white pigment may be mixed before the chroma is just apparently gone. These suggestions involve cut and try processes which are so laborious and tedious that they are probably rarely or never used.

Another serious objection is that pigments of different hues can not accurately be visually compared as to depth of tint. Their contrast in hue need not be great to be objectionable, for very slight variations in hue often make decisions of relative strengths unreliable. Thus, in judging between a yellow and an orange yellow, several observers might differ as to which is the stronger. 
A comparison of the tinting strengths of different pigments at various dilutions with a standard white, adjudged by our present methods, may reveal another real objection, the variability of tinting strengths in their relative order. In Figure 1 are plotted for two chrome yellow pastes two series of tints made by rubbing out each with zinc oxide in varying proportions. The brightnesses of the tints are plotted as ordinates and the compositions of the pigment mixtures as abscissas. Chrome yellow $A$ was a good grade of medium chrome yellow; $B$ was a dark chrome yellow selected because it was known to be somewhat coarse. Observe that the curves cross. If plotted to infinity, 100 per cent yellow, they would end at 33.4 per cent brightness for $B$, and at 46.4 per cent for $A$. On the assumption that the

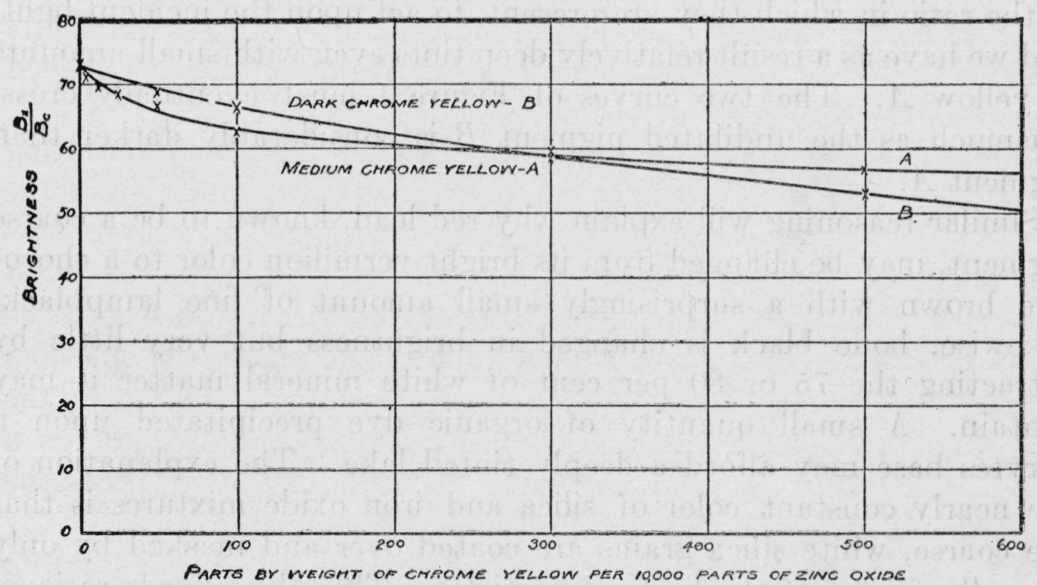

FIG. 1.-Brightness-composition curves, for two series of yellow tints, illustrating the crossing of these curves

darker the tint the stronger is the tinting substance (the validity of this assumption will be discussed later) which pigment, $A$ or $B$, is the stronger? One laboratory working at a ratio of 500 to 10,000 would report $B$ to be the stronger; another laboratory working at a ratio of 100 to 10,000 would report $A$ to be the stronger. The answer depends solely upon the proportion of white to yellow pigment.

The explanation of this variability in relative tinting strengths lies in the relative grain sizes of the white and of the yellow pigments. Zinc oxide is a sublimed product composed of very minute grains. Chrome yellows are precipitated products which may or may not be very fine. As a powder is ground increasingly fine, the surface of a constant weight becomes increasingly great, and the more surface that may be exposed to incident light the greater will be the tinting strength. Furthermore, as pointed out by Briggs, ${ }^{1}$ the possible tendency of the small particles to adhere to the surfaces

1 T. R. Briggs, J. Phys. Chem., 22, p. 223; 1918. 
of the larger ones may be another factor of importance. The average particle size of the chrome yellow $B$ was probably comparatively great, and the zinc oxide particles tended to coat over the larger yellow surfaces, and thus to produce an appearance approximating that of the white coating material. The resulting degree of apparent whiteness would, of course, depend on other factors, such as the opacities of the two pigments and their powers to diffuse light. As more and more chrome yellow is added, it is obvious that more yellow surface will be exposed with an increase in the proportion of yellow light reflected. If the particles of chrome yellow $A$ are sufficiently small, little or no coating by the fine white may occur. Thus, the yellow and white particles would have equal opportunity, in the ratio in which they are present, to act upon the incident light, and we have as a result relatively deep tints even with small amounts of yellow $A$. The two curves of Figure 1 must eventually cross, inasmuch as the undiluted pigment $B$ is considerably darker than pigment $A$.

Similar reasoning will explain why red lead, known to be a coarse pigment, may be changed from its bright vermilion color to a chocolate brown with a surprisingly small amount of fine lampblack. Likewise, bone black is changed in brightness but very little by extracting the 75 or 80 per cent of white mineral matter it may contain. A small quantity of organic dye precipitated upon a barytes base may afford a deeply tinted lake. The explanation of the nearly constant color of silica and iron oxide mixtures is that the coarse, white silica grains are coated over and masked by only a small amount of the fine red particles. This also affords rational ground for the preference for coarse mineral blacks over fine lampblacks when the tinting strengths of white pigments are used as indications of their hiding powers. For certainly, in such a measurement, the white should coat and hide the black rather than the reverse.

\section{DISCUSSION OF TINTING STRENGTH}

The American Society for Testing Materials has adopted the following definition of tinting strength: "The power of coloring a given quantity of paint or pigment selected as a medium standard for estimating such power." This definition is in accord with the common conception of color strength among paint men generally, but it is not definite enough to permit its use as a basis for a measurement of tinting strength.

What is meant by the term "power of coloring"? Offhand, one might suggest "power to change the degree of color." However, this definition fails when one considers the American Society for Testing Materials definition for color, "A generic term referring 
inclusively to all the colors of the spectrum, white and black, and all the tints, shades, and hues which may be produced by their admixture." According to this definition, a gray, a red, and a pink are three separate and distinct colors, and one may not be said to possess a greater degree of color than another.

It is generally understood that there are three attributes by which color is characterized. These are hue, brilliance, and saturation. According to the latest nomenclature these terms belong to the psychological side of the phenomenon of color, the corresponding stimulus terms being, respectively, dominant wave length, brightness, and purity. By hue is meant that attribute of certain colors in respect of which they differ characteristically from the gray of the same brilliance. ${ }^{2}$ In this quality, for example, red differs from a green. By brilliance is meant that attribute of any color in respect of which it may be classed as equivalent to some member of a series of grays ranging between black and white. ${ }^{2}$ In brilliance dark green differs from a light green. By saturation is meant that attribute of all colors possessing a hue which determines their degree of difference from a gray of the same brilliance. ${ }^{2}$ In saturation a pure spectral red differs from a grayish red.

These three attributes can be independently varied. What then occurs when a chromatic pigment is used for "coloring" a white in the production of a tint? Does only one attribute change, or do some two, or all three? And what effect does any change that may occur have upon the phenomenon we understand as tinting?

Considering, first, possible changes in hue when a chromatic pigment is diluted with a white, such changes do occur and are not necessarily due to any lack of perfect neutrality of the white, although the so-called whites are invariably not strictly neutral but incline to yellowish, bluish, etc. When a chromatic pigment is diluted with a white one, light rays internally transmitted within a paint film or pigment mass will have a shorter mean path through selectively absorbing grains before reflection into the atmosphere, because of the interspersion of the highly diffusing and refracting white grains. Merwin ${ }^{3}$ has pointed out that one effect of this will be the shifting of the dominant radiation in the direction of maximum spectral absorption. Merwin has shown also, that because of the preferential scattering of light by fine particles the presence of very fine white pigment may cause a migration of the hue toward the violet end of the spectrum.

Enough has been said to show that real changes in hue often do occur when chromatic pigments are mixed with white pigments to

${ }^{2}$ J. Opt. Soc. Am. and Rev, Sci. Inst., 6, No. 6, p. 534; August, 1922.

3 "Optical properties and theory of color of pigments and paints," Proc. Am. Soc. Test. Mats., 17, Pt. II, p. 496; 1917. 
form tints. Do these changes play any part in our conception of tinting? Hue is the name for a psychological sensation which so differs from the sensation of gray as to permit such designation as reddish or yellowish. It must be sharply distinguished from saturation which is the sensation produced by the apparent intensity of the hue. A color either evokes a hue or it does not. Thus, a red and a pink may differ in saturation but be of the same hue, and it can not properly be said that one possesses more hue than the other. The physical measure of the hue of a yellow tint might be $580 \mathrm{~m} \mu$, of a red $680 \mathrm{~m} \mu$. But the red pigment would not have more strength than the yellow by virtue of its being red, or vice versa. Hue and tinting strength are not interdependent qualities. It is true that two observers might argue vehemently over the relative depths of two tints, a bluish green and a yellowish green. The differences in hue would prejudice the individual opinions, but this is due to a peculiarity of the psychological method of judging. The observers make their judgments from different points of view, each subconsciously preferring a different hue and probably basing his .opinion largely upon the saturation of the colors with respect to this preferred hue. Hue desired is a matter of individual taste, and color and coloring efficiency must be considered separately.

Let us now observe how saturation and brilliance change during the process of "coloring" a white pigment in the production of a tint. It is obvious that both of these attributes do change. In the first place, a black pigment added to a white certainly produces a gray of intermediate brilliance. In the second place, any given color may be considered a mixture of pure color with gray, and the saturation of the color by definition depends upon its departure from gray. Make a tint, then, by adding to any given chromatic color an additional proportion of gray and the saturation will necessarily be diminished.

The important question, now, is whether a tint is formed from the white by admixture with another color because of an increase of saturation, because of a reduction of brilliance, or because of a change in both of these attributes.

Let us consider for the moment only achromatic pigments; that is, blacks and whites, evoking no hue and, therefore, possessing zero saturation. Any change on mixing these colors will be in the main a change in brilliance. Gardner, in "Paint Technology and Tests," says of mineral black: "It contains a comparatively low percentage of carbon, and consequently has low tinting value." Langton (London), in "Blacks and Pitches," says of bone blacks: "Acidwashed black has a very deep, black colour, and in consequence of its fine state of division a great deal of colour strength." Toch, in "Chemistry and Technology of Paint," says in regard to mineral 
blacks: "As they possess very little tinctorial power it is more advantageous to use a 200 -mesh silica tinted with lampblack." The point to which attention is called in each of these quotations is that the blacks are considered to possess tinting or color strength. The same usage is general throughout the literature, and any definition or discussion of tinting strength must conform to this generally accepted opinion that blacks do possess tinting strength by virtue of their power to decrease the brilliance of a white.

In the case of tinting with chromatic pigments both the brilliance and the saturation usually change. The conditions under which tints can be made with change in saturation but without change in brilliance must be specially selected. Consider Figures 1 and 13. In these figures the physical analogues of brilliance and saturation have been employed; namely, brightness and purity, respectively. Brilliance bears a linear relationship to the logarithm of the brightness according to Fechner's law, and whatever the relationship between saturation and purity this correlative use of the terms is permissible for our immediate discussion in which colors of nearly the same dominant wave length are being considered. It may be observed from Figure 1 that at about the ratio of 300 to 10,000 the two chrome yellow tints have the same brightness. From Figure 13, however, we observe that at this ratio the medium yellow has a very much higher purity than the dark. Show these two particular tints to color men with the question, "Which pigment is the stronger tinter?" and the typical answer would be, "Well, they differ a little in tone, but I'd say $A$ is the stronger." Undoubtedly $A$, the medium chrome yellow, should be considered to possess the greater tinting strength. Of course, there is only one point on the two brightness-composition curves where the two would coincide, but in this special event there would be a difference in the strength of the two tints which would depend solely upon purity or saturation. Thus, it must be admitted that saturation as well as brilliance is a factor in the determination of tinting strength.

Indeed, it may be argued that, aside from the case of black pigments which are admittedly used in tinting to diminish brilliance, saturation alone is the factor upon which tinting depends. According to these arguments, if a painter adds a red pigment to a white to obtain a tint he does so for the sole purpose of producing a product which will appear reddish. The accompanying decrease in brilliance because of absorption of blue and green rays is not the painter's aim, but is, indeed, a necessary evil attending his method of producing a red color. Had he some other practical way to produce a red tint without lessening the brilliance he probably would utilize this alternative method. 
However, $i_{i}$ is generally true that, when a white is tinted with any other color than black, both brilliance and saturation change. It is possible that these two attributes are mentally combined into one sensation which has been called the depth of tint. It does seem, however, that if there is any psychological tendency toward combination, it is certainly not perfect, for of two tints of the same hue varying in both brilliance and saturation it is extremely difficult to select the deeper. Accordingly, it seems necessary that the two attributes be considered separately and a distinction made between the power of a pigment to alter the brilliance of a white and its power to alter the saturation of a white.

The relationship between brightness and brilliance has been fairly well determined. Fechner's expression for this relationship is $S=A \log I+c$, where $S=$ the brilliance sensation, $I=$ the brightness stimulus, and $A$ and $c$ are equating constants. Colorimetric purity is a physical quantity defined as the ratio

$$
\frac{B_{\Lambda}}{B_{\Lambda}+B_{\mathrm{w}}}
$$

where $B_{\Lambda}$ is the brightness of the homogeneous constituent and $B_{\mathrm{w}}$ is the brightness of the white light in a mixture of homogeneous and white light which matches the particular color in question. As such a physical quantity, purity can be numerically stated and easily treated. On the other hand, the relationship between purity and saturation is not thoroughly known, and it is not yet possible to represent saturation by a numerical designation. This lack of information abruptly checks our logical derivation of a means of expressing tinting strength in the most suitable and desirable way, on the basis of the degree of saturation possessed by the tint.

\section{EXPERIMENTAL DATA}

In this study of tinting considerable experimental work has been carried out in the endeavor to ascertain just how the brightness and the purity are altered when the proportion in which a white pigment is mixed with a pigment of another color is varied. Many series of tints were made up from one chosen standard white, each series being made from a different pigment in combination with the white, and consisting of several steps, grading from the untinted white through darker and purer colors, to the undiluted test pigment. Each test pigment was ground on a roller mill or thoroughly rubbed out by muller and stone with sufficient bleached linseed oil to form a paste of rather thin consistency. The standard white was a good grade of zinc oxide ground on a roller mill to a paste containing 25 per cent of oil by weight. To form each tint small portions of these pastes were accurately weighed and mixed in the glass weighing bottle 
B. S. Journal of Research, RP7

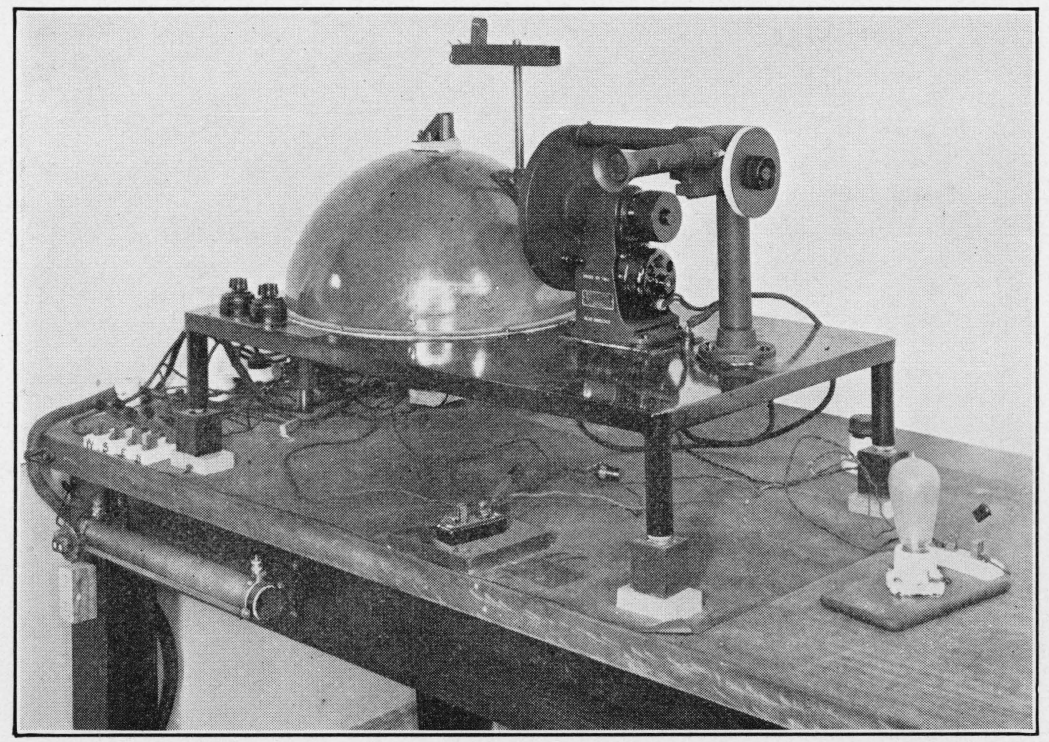

Fig. 2.-Spectrophotometer on which color measurements were made 
with a stirring rod. The resulting tinted pastes were prepared for the color measurement by incasing a layer thick enough to be opaque between selected glass microscope slides.

The color measurements were made with a spectrophotometer. The particular color analyzer used in this investigation is shown in Figure 2. From the data of the spectrophotometric curves, which are reproduced in Figures 3 to 11, inclusive, the color of each tint was computed in terms of its dominant wave length, purity, and relative

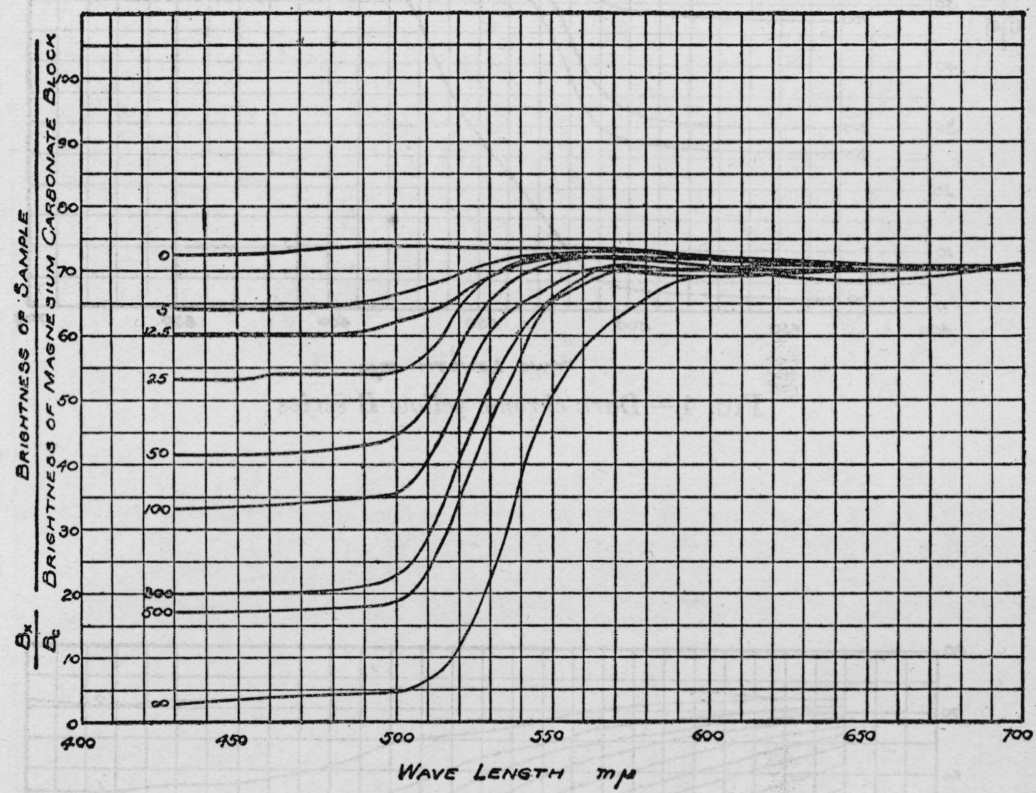

FIG. 3.-Medium chrome yellow series. Spectrophotometric curves for a zinc oxide paint paste, a medium chrome yellow paste, and seven tints made by their admixture in various proportions

The curve numbered 0 is for the zinc oxide; the curve $\infty$ is for the undiluted yellow; the curve 500 is for a paste made by mixing 500 parts of the chrome yellow pigment with 10,000 parts of the zinc oxide; etc., for the other curves.

brightness (reflection for sunlight). ${ }^{4}$ The relative brightnesses of the gray series were measured by means of a Martens photometer. ${ }^{5}$ This was preferred for this purpose to the spectrophotometer because of its greater simplicity and precision. In Figure 12 are plotted brightness-composition curves for several series, and in Figure 13 are plotted purity-composition curves. Each curve, beginning at the point representing the white, slopes away at a characteristic rate and approaches asymptotically the brightness or the purity, respectively, of the undiluted solid color.

4 Vid. Appendix for the method used to make these computations.

${ }^{5}$ Illustrated in B. S. Tech. Paper No. 306, p. 175; 1926.

$110118^{\circ}-28-2$ 


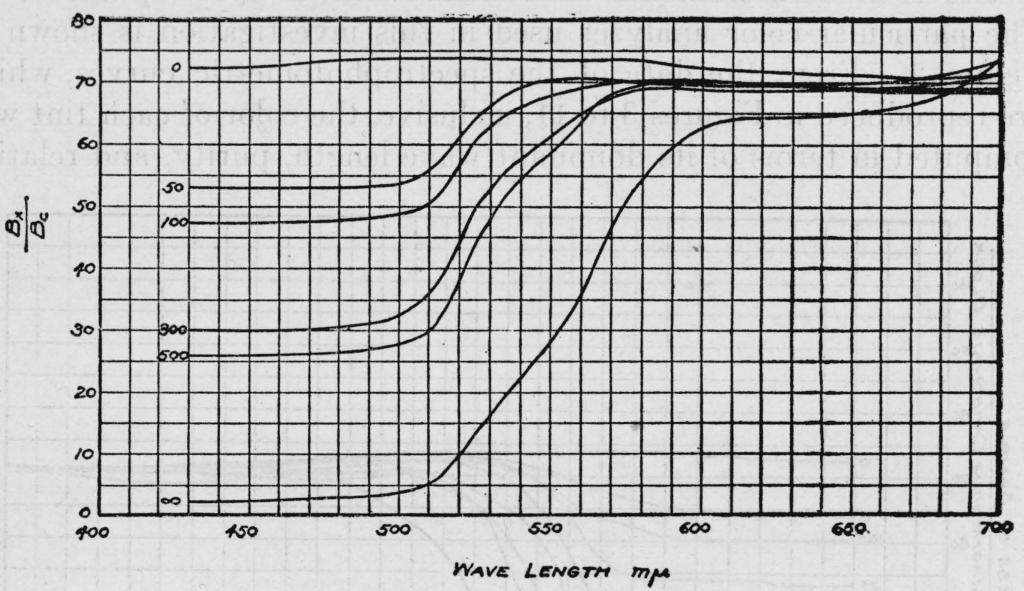

FIG. 4.-Dark chrome yellow $B$ series

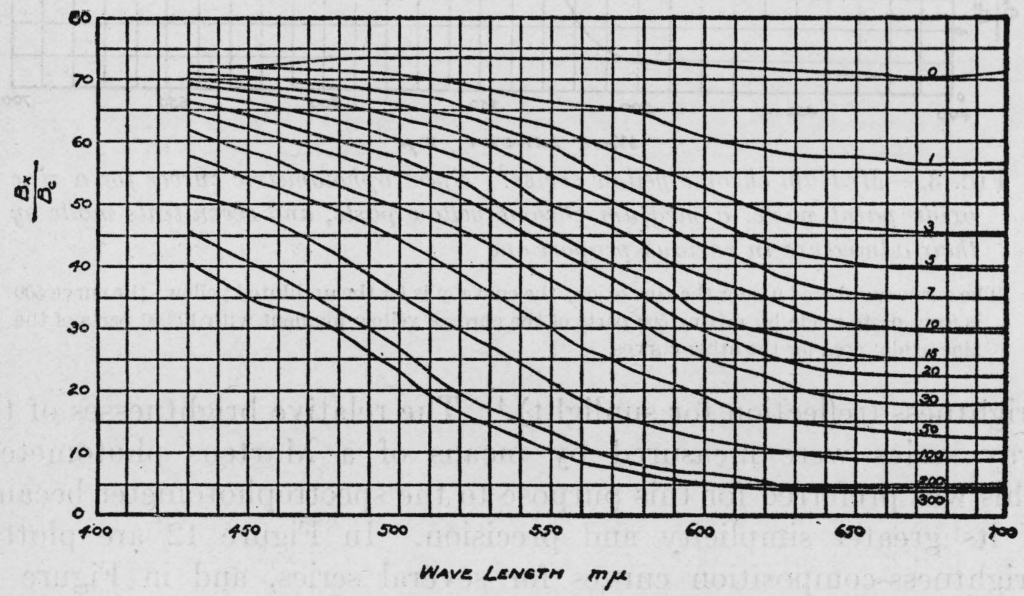

Fig. 5.-Prussian blue series 


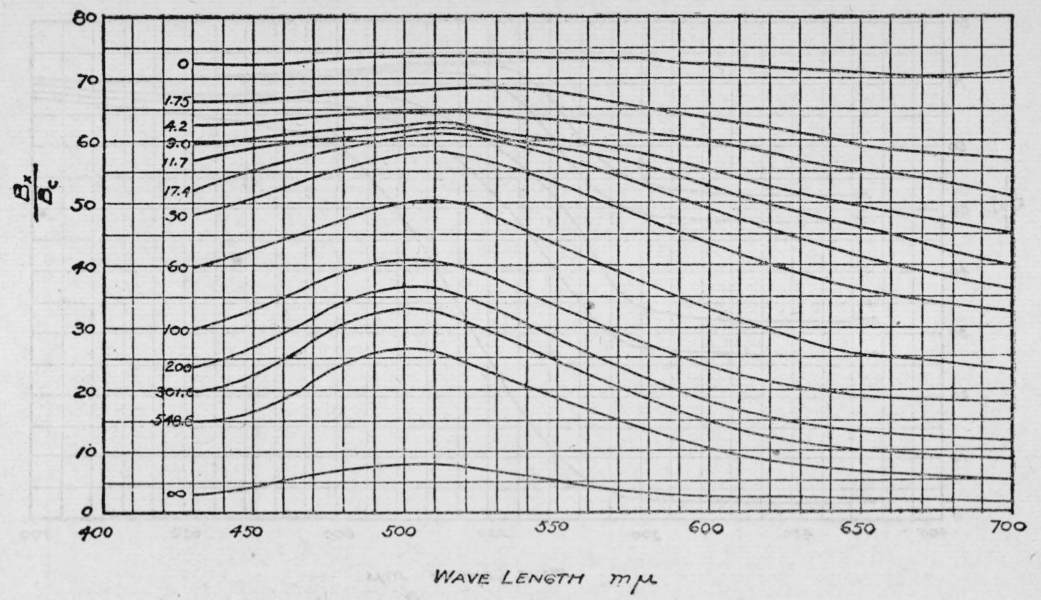

FIG. 6.-Chrome green (first determination) series

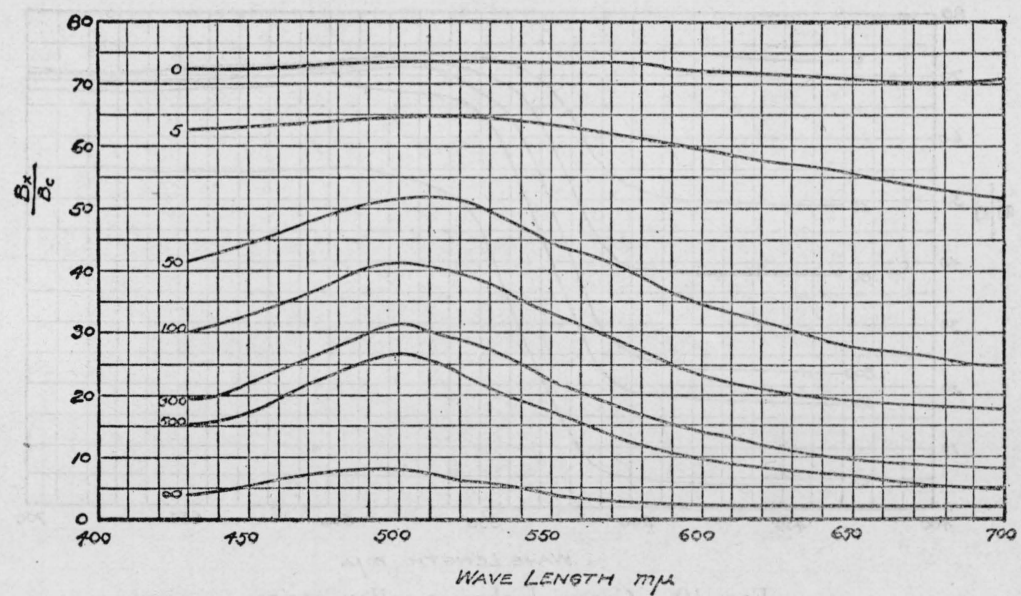

Fig. 7.-Chrome green (second determination) series

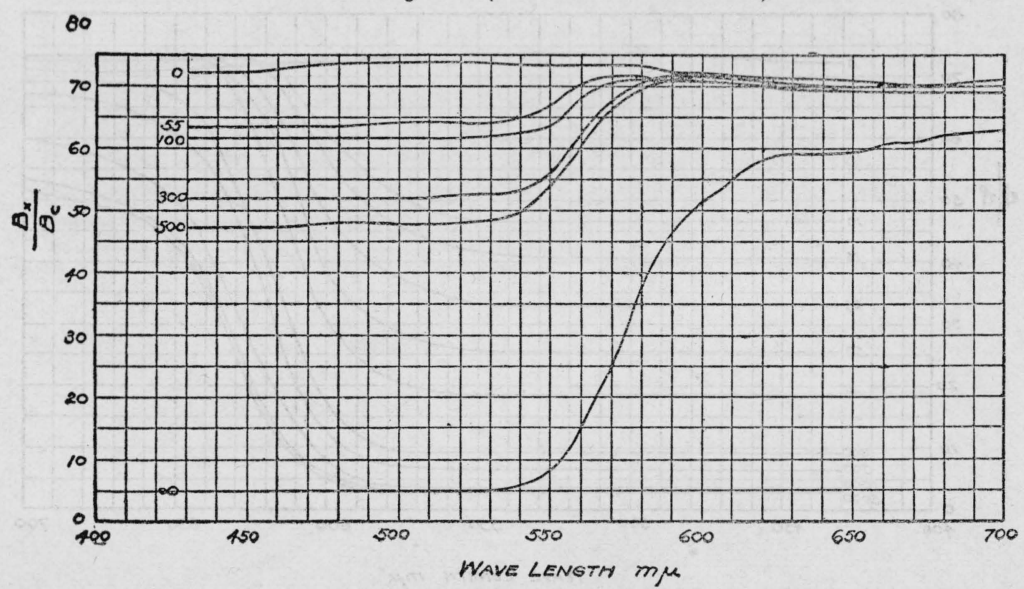

Fig. 8.-Red lead series 


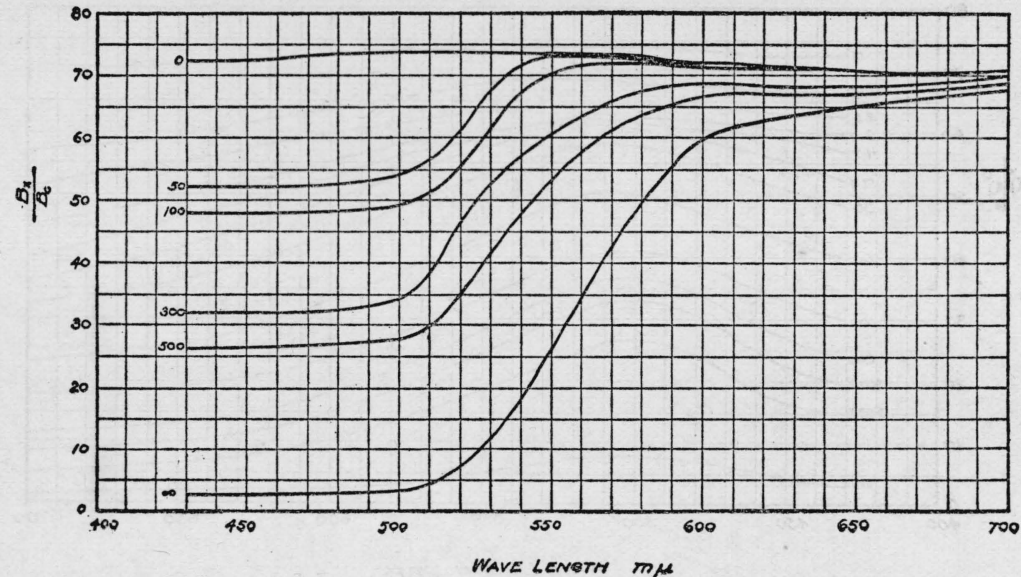

FIG. 9.-Dark chrome yellow series

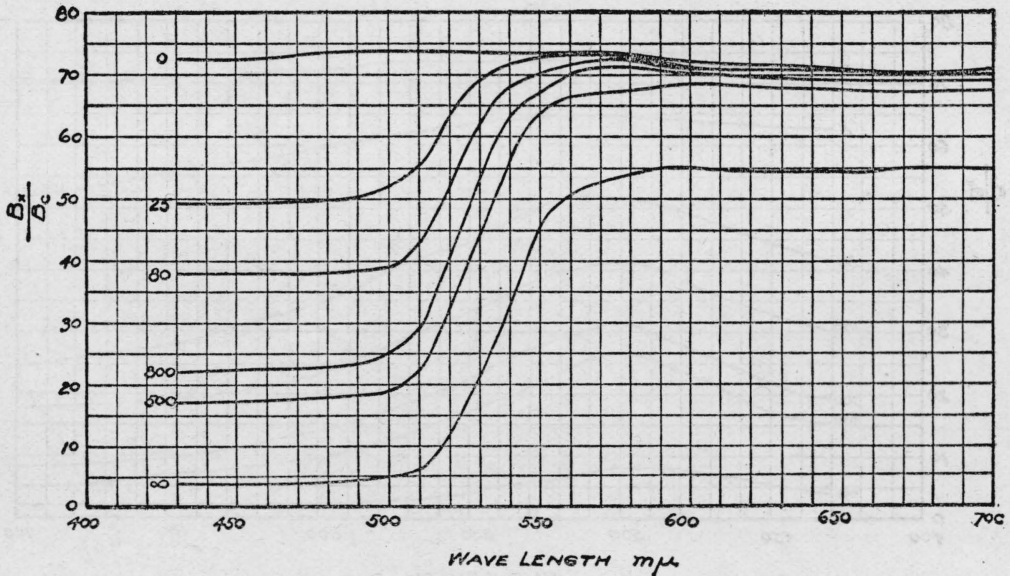

FIg. 10.-Greenish chrome yellow series

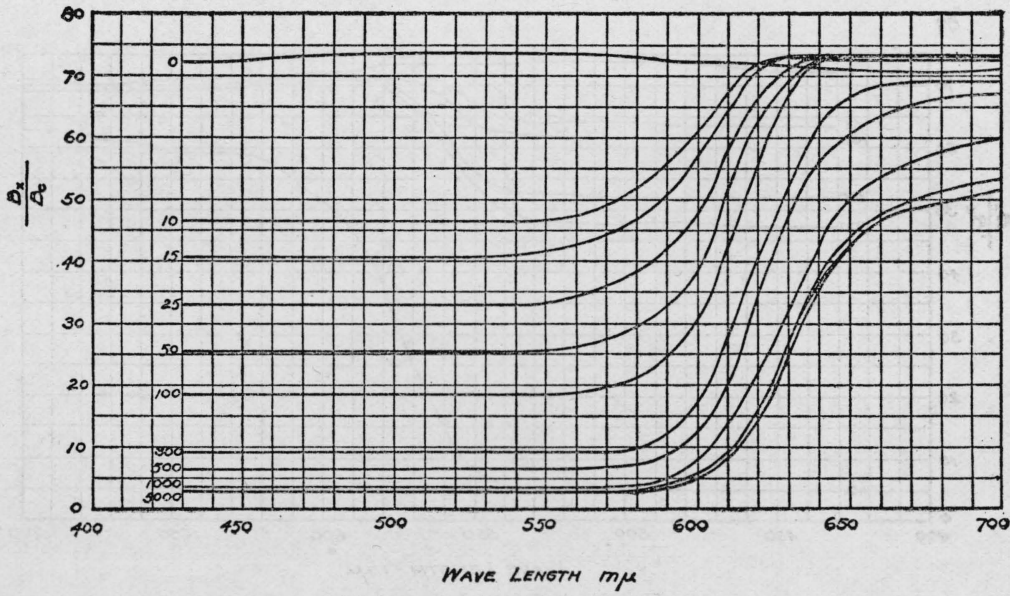

Fig. 11.-Para red series 
Inasmuch as the paint pastes were measured under glass slides, the brightness figures obtained were not true brightness values relative to a magnesium carbonate block for the pastes alone. To

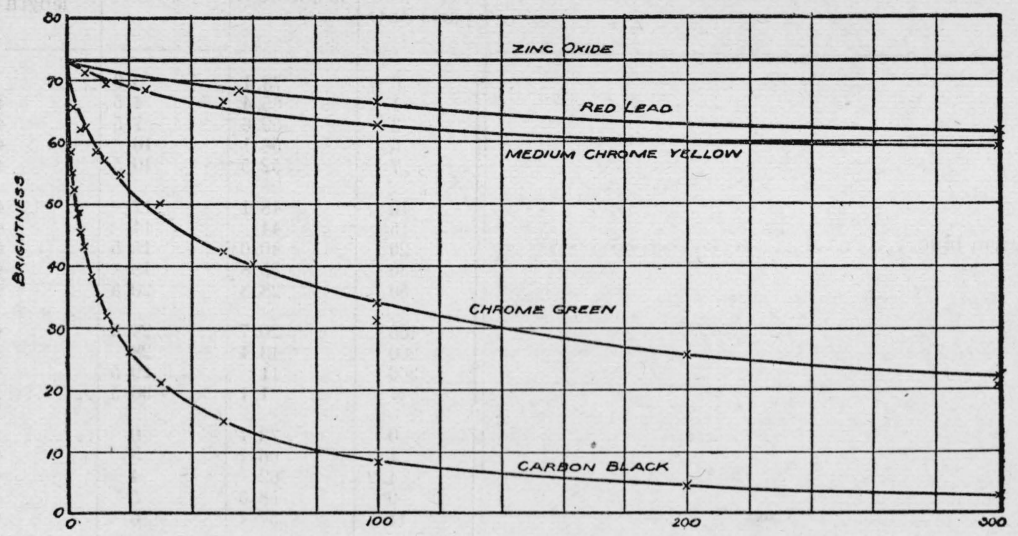

PAATS or WEIGHT OF TEOT PIGMENT PER 10,000 PAATO OF ZINC OXIDE

FIG. 12.-Typical brightness pigment composition relationship curves for several series of tints

obtain these true brightnesses a correction would have to be applied for the proportion of the light lost on account of the presence of the cover glass. However, this correction is the same for all observa-

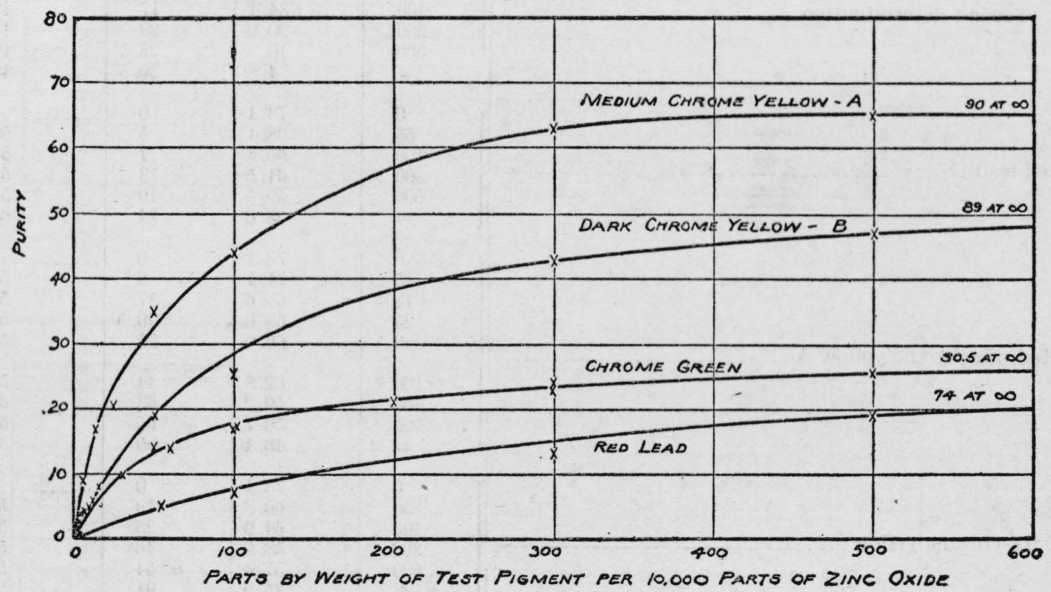

FIG. 13.-Typical colorimetric purity-composition relationship curves for several series of tints

tions and cancels out during subsequent calculations. It has, therefore, not been applied to the brightness figures of the data which will follow. 
TABLE 1.-Tint composition and color data

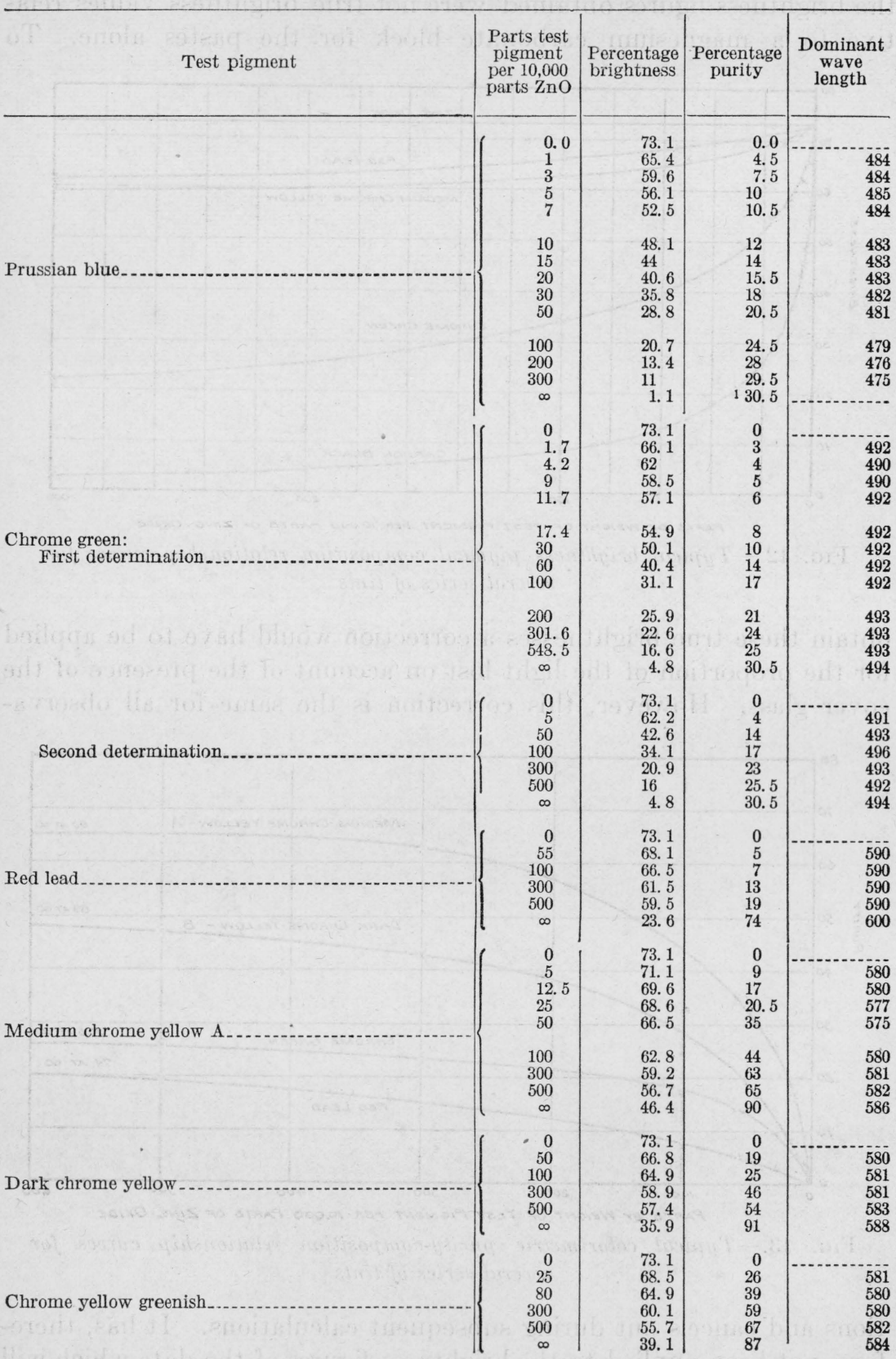

1 Assumed. 
TABLe 1.-Tint composition and color data-Continued

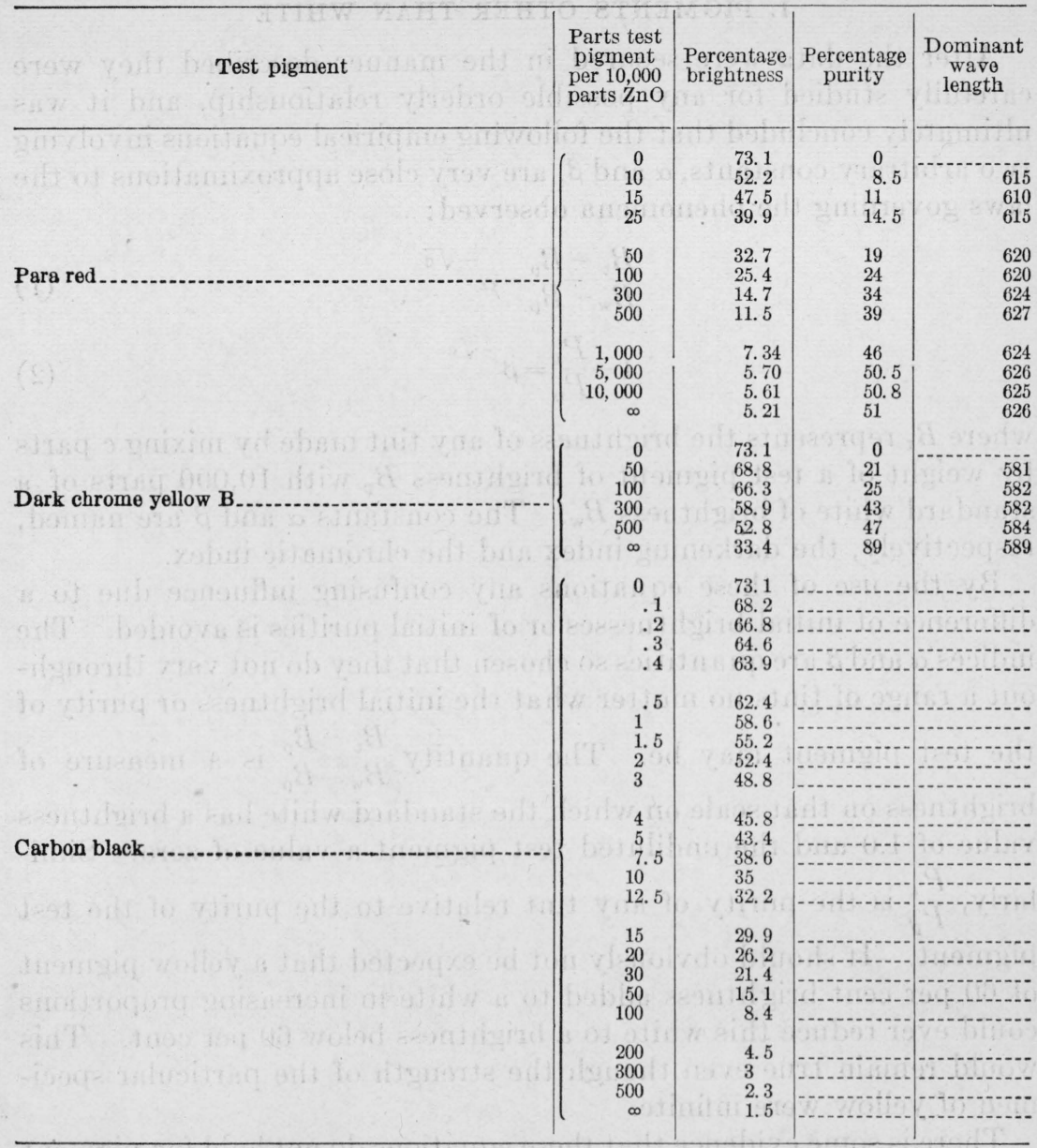

TABLE 2.-Pigment composition and brightness data for gray tints

\begin{tabular}{|c|c|c|c|c|c|c|c|}
\hline \multirow{2}{*}{$\begin{array}{l}\text { Parts of carbon black per } 10,000 \\
\text { parts of white pigment }\end{array}$} & \multicolumn{7}{|c|}{ Percentage brightness relative to magnesium carbonate block } \\
\hline & $\mathrm{TiO}_{2}$ & Titanox & $\begin{array}{l}\text { Litho- } \\
\text { pone }\end{array}$ & $\begin{array}{l}\text { White } \\
\text { lead }\end{array}$ & $\mathrm{ZnO}$ & $\mathrm{ZnS}$ & $\underset{\text { fixe }}{\text { Blane }}$ \\
\hline $\begin{array}{l}0.00 \\
0.25\end{array}$ & 76.9 & 79.1 & 78.3 & 76.6 & 76.2 & 77.7 & $\begin{array}{l}60.8 \\
38.8\end{array}$ \\
\hline 1.00 & & & & & & & 24.9 \\
\hline 2.47 & 60.6 & 53.8 & 52.3 & 48.3 & 50.8 & 58.9 & 15. 1 \\
\hline 10.72 & 45.9 & 36.2 & 34.5 & 28.4 & 32.3 & 43.5 & 4. 24 \\
\hline & 35.3 & 24.5 & 23.1 & 17.8 & 20.8 & 31.9 & 2. 29 \\
\hline 44.5 & 27.2 & 17.3 & 15.8 & 11.2 & 14.1 & 23.6 & 1. 77 \\
\hline 71.21 & 21.4 & 12.4 & 11.0 & 7.6 & 9.9 & 18.2 & 1. 63 \\
\hline 104. & 17.4 & 9.0 & 8.0 & 5.0 & & 14.1 & 1. 59 \\
\hline$\infty$ & 1.5 & 1.5 & 1.5 & 1.5 & 1.5 & 1.5 & 1.5 \\
\hline
\end{tabular}




\section{DISCUSSION OF DATA}

\section{PIGMENTS OTHER THAN WHITE}

After the data were secured in the manner described they were carefully studied for any possible orderly relationship, and it was ultimately concluded that the following empirical equations involving two arbitrary constants, $\alpha$ and $\beta$, are very close approximations to the laws governing the phenomena observed:

$$
\begin{aligned}
& \frac{B_{\mathrm{t}}-B_{\mathrm{p}}}{B_{\mathrm{w}}-B_{\mathrm{p}}}=\alpha^{-\sqrt{\mathrm{c}}} \\
& 1-\frac{P_{\mathrm{t}}}{P_{\mathrm{p}}}=\beta^{-\sqrt{\mathrm{c}}}
\end{aligned}
$$

where $B_{\mathrm{t}}$ represents the brightness of any tint made by mixing $c$ parts by weight of a test pigment of brightness $B_{\mathrm{p}}$ with 10,000 parts of a standard white of brightness $B_{\mathrm{w}}$. The constants $\alpha$ and $\beta$ are named, respectively, the darkening index and the chromatic index.

By the use of these equations any confusing influence due to a difference of initial brightnesses or of initial purities is avoided. The indices $\alpha$ and $\beta$ are quantities so chosen that they do not vary throughout a range of tints no matter what the initial brightness or purity of the test pigment may be. The quantity $\frac{B_{\mathrm{t}}-B_{\mathrm{p}}}{B_{\mathrm{w}}-B_{\mathrm{p}}}$ is a measure of brightness on that scale on which the standard white has a brightness value of 1.0 and the undiluted test pigment a value of zero. Similarly, $\frac{P_{\mathrm{t}}}{P_{\mathrm{p}}}$ is the purity of any tint relative to the purity of the test pigment. It should obviously not be expected that a yellow pigment of 60 per cent brightness added to a white in increasing proportions could ever reduce this white to a brightness below 60 per cent. This would remain true even though the strength of the particular specimen of yellow were infinite.

There is some evidence that these equations do not hold for pigment mixtures which are very close in color to the test pigment. However, we are here concerned with tints - that is, relatively light colors-and these empirical equations hold well within our experimental precision over the requisite range.

Transforming equations (1) and (2) to their logarithmic forms

$$
\begin{aligned}
& \log \frac{B_{\mathrm{t}}-B_{\mathrm{p}}}{B_{\mathrm{w}}-B_{\mathrm{p}}}=-\sqrt{c} \log \alpha \\
& \log \left(1-\frac{P_{\mathrm{t}}}{P_{\mathrm{p}}}\right)=-\sqrt{c} \log \beta
\end{aligned}
$$

and plotting $-\sqrt{c}$ against $\log \frac{B_{\mathrm{t}}-B_{\mathrm{p}}}{B_{\mathrm{w}}-B_{\mathrm{p}}}$ and $\log \left(1-\frac{P_{\mathrm{t}}}{P_{\mathrm{p}}}\right)$, respectively, straight lines result with their slopes equal to the logarithms of $\alpha$ and $\beta$, respectively. 
In Figures 14 and 15 these straight lines have been plotted. The values of $\alpha$ and $\beta$ for different pigments lie between 1 and infinity. The greater the value of $\alpha$ the greater is the darkening strength. The greater the value of $\beta$ the greater is the chromatic strength. Thus, it may be observed from Figures 14 and 15 that red lead, very weak in tinting strength, has low values for both $\alpha$ and $\beta$. Para red, on the other hand, ordinarily a powerful pigment, has considerably larger values for its darkening and chromatic indices.

TABLe 3

\begin{tabular}{|c|c|c|c|c|c|}
\hline Test pigment & $\begin{array}{c}\text { Darken- } \\
\text { ing } \\
\text { index }\end{array}$ & $\begin{array}{l}\text { Chro- } \\
\text { matic } \\
\text { index }\end{array}$ & Test pigment & $\begin{array}{c}\text { Darken- } \\
\text { ing } \\
\text { index }\end{array}$ & $\begin{array}{l}\text { Chro- } \\
\text { matic } \\
\text { index }\end{array}$ \\
\hline $\begin{array}{l}\text { Red lead } \\
\text { Dark chrome yellow } \mathrm{B} \\
\text { Dark chrome yellow } \\
\text { Greenish chrome yellow } \\
\text { Medium chrome yellow }\end{array}$ & $\begin{array}{l}\text { 1. } 014 \\
\text { 1. } 026 \\
\text { 1. } 027 \\
\text { 1. } 029 \\
\text { 1. } 044\end{array}$ & $\begin{array}{l}\text { 1. } 012 \\
\text { 1. } 036 \\
\text { 1. } 040 \\
\text { 1. } 068 \\
1.064\end{array}$ & $\begin{array}{l}\text { Chrome green } \\
\text { Para red } \\
\text { Prussian blue-_. } \\
\text { Carbon black }-. . \\
\end{array}$ & $\begin{array}{l}\text { 1. } 084 \\
1.136 \\
1.139 \\
1.266\end{array}$ & $\begin{array}{l}1.083 \\
1.067 \\
1.180\end{array}$ \\
\hline
\end{tabular}

In Table 3 are summarized the numerical indices found for various pigments. It must be understood that these figures are in no way absolute, inasmuch as they depend upon the particular white pigment arbitrarily selected as the standard. It should be emphasized, also, that these indices are measured on a scale determined by the properties of the particular pigment, and therefore do not represent what might properly be considered absolute tinting strength even with the one white pigment. This absolute tinting strength, dependent upon the actual depth of a tint, is not constant for any one pigment, but varies with the concentration used. These facts are well illustrated by the curves of Figure 1 in comparison with those for the same pigments in Figure 14. The medium chrome yellow pigment has a larger darkening index than the dark yellow, so that its graph as plotted in Figure 14 on the basis of equation (3) falls distinctly below that for the darker pigment. The significance of this is that a small quantity of the lighter pigment has a relatively large effect and carried the tint much further toward the depth of color shown by the pigment itself, although the absolute effect may not be materially different from that given by an equal amount of the darker pigment. Furthermore, despite the fact that all of these eight pigments in Table 3 fall, when their strength is estimated in actual practice, in the same order as listed in the table, we are not at liberty to draw comparisons between pigments of different hues from the values of their chromatic indices. Thus, if it were possible to have a coarse Prussian blue of the same chromatic index as a medium chrome yellow, tints made from the same proportions of the two pigments mixed with a white, although of the same colorimetric purity, would not appear equally saturated and, therefore, 


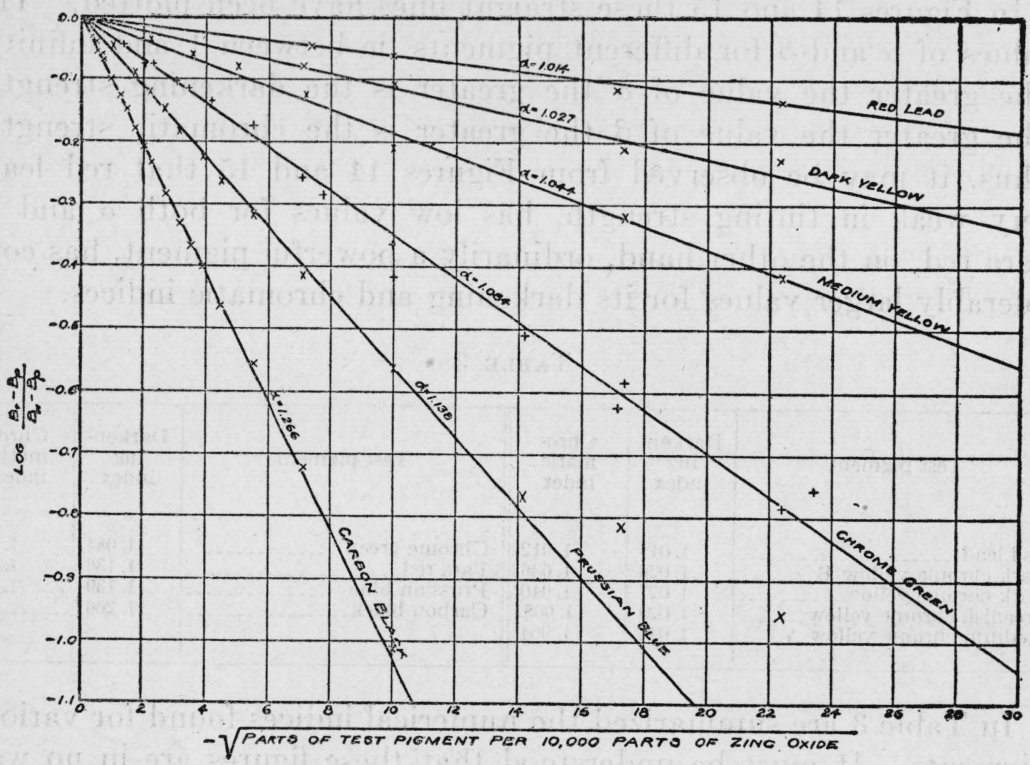

FIG. 14.-Diagrammatic illustration of a logarithmic relationship between the brightness of a tint and its pigment composition

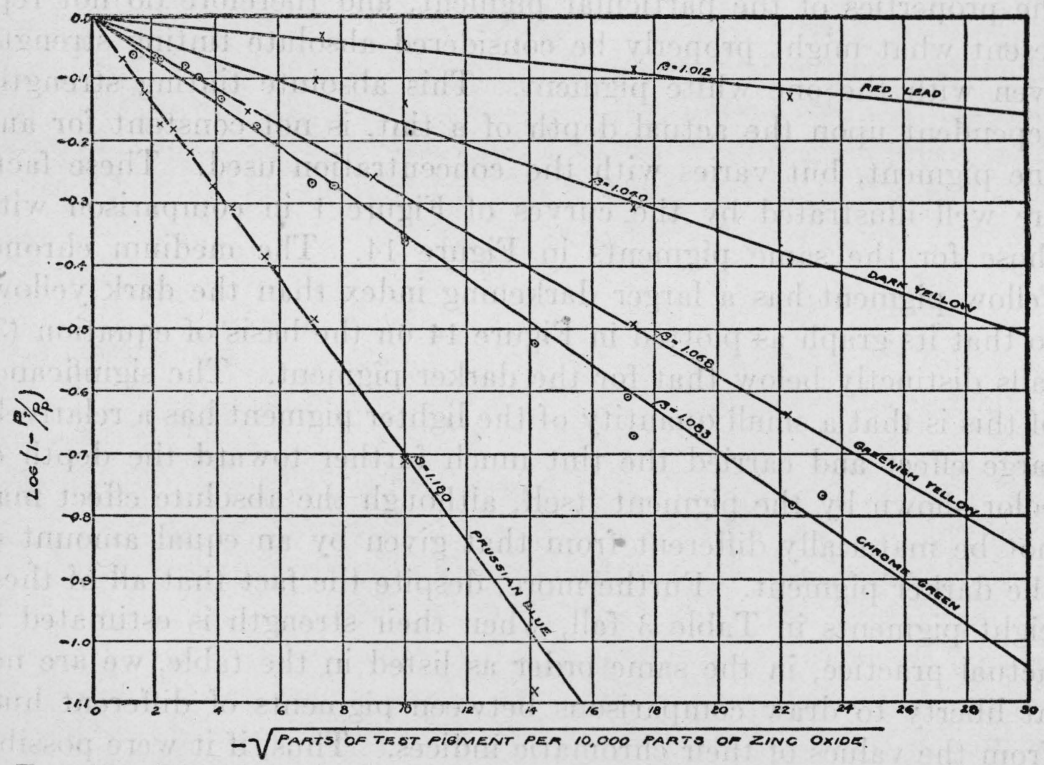

FIG. 15.-Diagrammatic illustration of a logarithmic relationship between the colorimetric purity of a tint and its pigment composition 
not of the same depth of tint. The blue might be highly saturated and deeply tinted at a degree of purity at which the yellow might appear nearly white. It is, however, certainly permissible to contrast together all pigments of the same hue as all ultramarines or all yellow ochers, according to their chromatic indices. As it is probably never in actual practice required to compare as to tinting strength pigments of widely different hues, this restriction is perhaps after all not particularly stringent.

\section{WHITE PIGMENTS}

Heretofore attention has been confined to pigments other than whites. There exists a reason for this discrimination. A knowledge of the tinting strength of white pigments is arrived at by quite another process, differentiated from that used with other pigments by two important distinctions. One is that with whites a dark pigment must be used as the standard. The other is that in testing whites a large quantity of the test pigment is used with a small quantity of the standard, a procedure just the reverse of that employed for testing blacks and chromatic pigments. Thus, in estimating the tinting strength of a white lead a large portion is mixed as a paste with a small amount of the standard black. The depth of shade of the resulting gray is dependent upon that property of the standard black which we have called darkening strength. From the apparent darkening strength (apparent inasmuch as the actual darkening strength is that obtained only with the standard white) is derived by inference the power of the white to brighten. The two bear a reciprocal relationship to one another; the less the apparent darkening strength the greater the brightening strength. Thus, there is justification in defining $\gamma$, the index of brightening strength of white pigments as $\frac{1}{\alpha}$, where $\alpha$ is the darkening index of the black. As a result the index of brightening strength of a white pigment may be calculated from the following equation:

$$
\frac{B_{\mathrm{t}}-B_{\mathrm{p}}}{B_{\mathrm{w}}-B_{\mathrm{p}}}=\gamma^{\sqrt{\mathrm{c}}}
$$

where $B_{\mathrm{t}}$ represents the brightness of any tint made by mixing $c$ parts by weight of the standard black of brightness $B_{\mathrm{p}}$ with 10,000 parts of the white test pigment of brightness $B_{\mathrm{w}}$. The constant, $\gamma$, is named the brightening index. $\gamma$ varies between zero and unity. The greater the value of $\gamma$ the greater will be the brightening strength.

Transposing equation (5) to its logarithmic form, $\log \frac{B_{\mathrm{t}}-B_{\mathrm{p}}}{B_{\mathrm{w}}-B_{\mathrm{p}}}=\sqrt{c}$

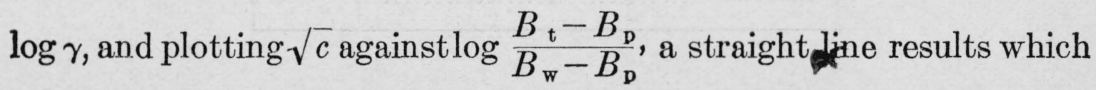


passes through the origin with a slope equal to $\log \gamma$. In Figure 16 several of these lines for various white pigments when mixed with a standard paste of carbon black have been plotted. Observe from Figure 16 how closely the experimental points fall upon the straight lines. No claim is made that the relationship should naturally and theoretically be linear. Indeed, a prolongation of these curves will reveal an actual upward curvature. What is claimed, however, and substantiated by Figure 16, is that the linear relationship is a very close and serviceable approach when confined to the realm of tints,

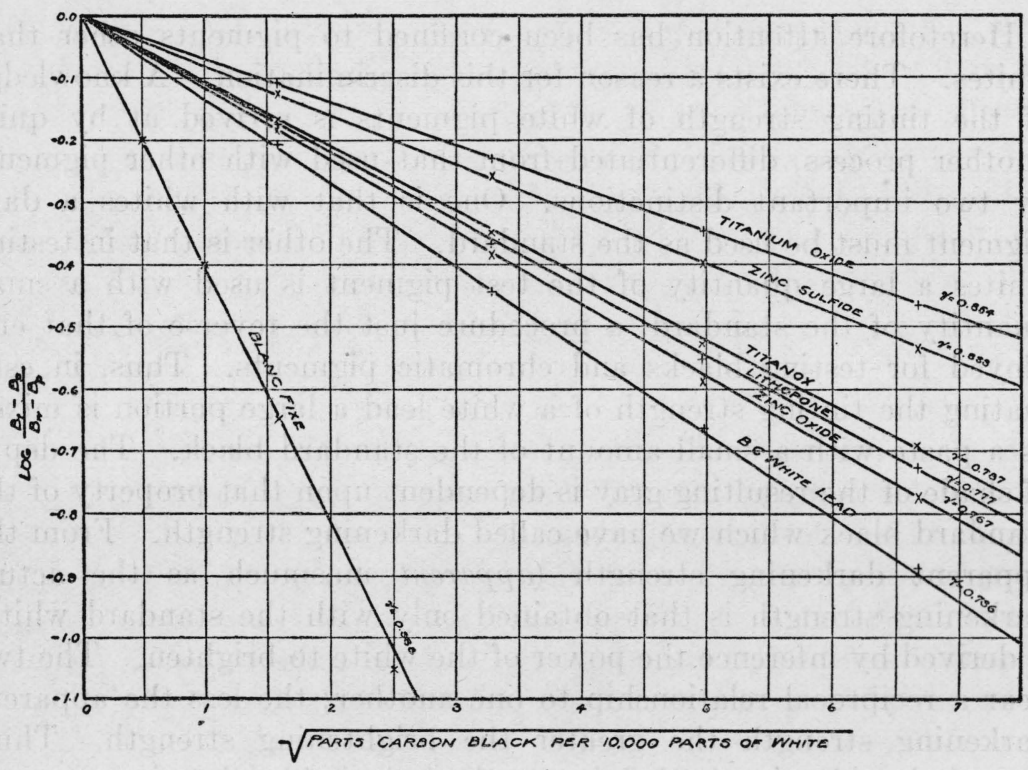

FIG. 16.-Diagrammatic illustration of a logarithmic relationship between the brightness of a gray tint and its constituent parts of black and white pigments

which as a specific and liberal approximation for this purpose may be considered to be above 20 per cent brightness. In Table 4 are summarized the numerical indices for individual samples of various white pigments.

TABLE 4

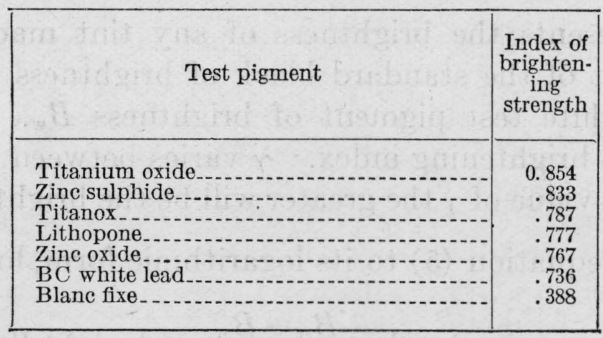


The data in Table 4 and Figure 16 were obtained by mixing the various white pastes with a standard paste of carbon black. These pastes, both the whites and the black, had been prepared for other purposes by grinding the pigment on a roller mill not with linseed oil, but with a colorless nondrying mineral oil.

To illustrate the method for evaluating the constants of equations (1), (2), and (5), let us consider a concrete example. The index of chromatic strength of a given chrome green is to be measured. Equation (2) containing $\beta$, the chromatic index, is $1-\frac{P_{t}}{P_{p}}=\beta^{\sqrt{c}}$. This particular sample of chrome green pigment has a purity of 30.5 per cent as measured spectrophotometrically. $P_{\mathrm{p}}$ then is 0.305 . A green tint is made by rubbing out some small amount of the green with a large amount of the standard white; say, 100 parts by weight of the green with 10,000 parts of the white. Quantity $c$ is then 100 . The purity of this tint, $P_{\mathrm{t}}$, is measured and found to be 17 per cent. Equation (2) becomes

$$
1-\frac{0.17}{0.305}=\beta^{-\sqrt{100}}
$$

with $\beta$ as the only unknown. Solving, $\beta=1.085$. This value is recorded for comparison with those of other chrome green pigments previously or subsequently tested.

Incidental to the subject of tinting strength, it is here interesting to note that equations (1), (2), and (5) provide means for predicting the purity and brightness that may be expected when two pigments, one of them white, are mixed in known proportions. Thus, assuming the index $\beta$ to equal 1.085 , as found above, what would be the purity of a tint composed of 200 parts of the same green with 10,000 of the white? Equation (2) becomes

$$
1-\frac{P_{t}}{30.5}=1.085^{-\sqrt{200}}
$$

$P_{t}$, the purity of the tint, is readily calculated to be 20.9 per cent. Actual measurements gave an observed value of 21 per cent for the purity. This is, perhaps, the first time that any attempt at precalculating the result of mixing pigments has been even approximately successful.

\section{APPENDIX}

By means of the spectrophotometer the spectral distribution of reflected light may be obtained in the form of a graph in which the portion of the incident light reflected has been plotted against wave length or frequency. The procedure that has been used in this inves- 
tigation for converting a spectrophotometric curve into color specifications in terms of dominant wave length, purity, and brightness, is based upon the data given in the report of the Colorimetry Committee of the Optical Society of America, 1920-21. ${ }^{6}$ For the purpose of showing how the computed values of brightness and purity were obtained the procedure will here be briefly presented.

When equal amounts of energy fall upon the retina of the eye as light of different wave lengths, quite different light intensities are experienced. This is because the human eye is not uniformly sensitive over the entire spectrum of radiant energy. In the ultra-violet the eye discerns no light whatever. Above this visibility rapidly mounts to a maximum in the greenish yellow and then falls again to zero in the infra-red. Consequently, when the brightness of a color is to be calculated from the spectrophotometric curve which represents the color, the relative values of visibility of the different wave lengths must be considered. A peak in the ultra-violet portion of the curve would add nothing to the brightness; a peak in the blue or red would add less than one in the green or yellow.

Furthermore, the brightness of a color is dependent upon the quality of the illuminating light. Thus, an orange might be very bright in sunlight but quite dark when viewed under a lamp deficient in yellow and red rays. Accordingly, in stating the brightness of a color, the character of the illumination must necessarily also be stated. White light is the common light for illumination, and brightness figures are usually calculated with reference to white light, which, for this purpose, has been specified as average noon sunlight.

A magnesium carbonate block has been found to reflect incident light almost nonselectively. Thus, the intensities of the various wave lengths of light reflected from a magnesium carbonate surface bear practically the same ratios to one another as they did in the incident light. Moreover, magnesium carbonate in air is one of the brightest of substances. A magnesium carbonate block makes, therefore, a very serviceable standard with which to compare samples in color examination. Observations with the spectrophotometer give for each wave length observed the ratio of the light reflected from the surface under examination to that reflected from magnesium carbonate; and a curve representing these ratios for wave lengths throughout the spectrum is the spectrophotometric or spectral reflection curve.

Considering one particular wave length, the greater its visibility, the greater the intensity of the incident light, and the greater the portion of the incident light reflected (the reflection factor) the greater will be the sensation of brightness caused by the reflected ray of that wave length. The contribution of that wave length to the

6 J. Opt. Soc. Am. and Rev. Sci. Inst., 6, 6, p. 527; August, 1922. 
brightness of the color may, therefore, be represented by VIR, product of the visibility of the particular wave length under consideration $(V)^{7}$, the intensity of that wave length in white light $(I)^{8}$ and the reflection factor $(R)$. If we take the reflection factor of the magnesium carbonate block as a unit, $R$ is given directly by the spectral reflection curve. Values of the product VI prepared from tables given in the report of the optical society previously referred to and expressed in arbitrary units are given in Table 5.

TABLE 5.-Spectral distribution of the visibility $\times$ intensity product for average noon sunlight

\begin{tabular}{|r|r|r|r|r|r|}
\hline $\begin{array}{c}\text { Wave } \\
\text { length }\end{array}$ & $\begin{array}{c}\text { Visibility } \\
\times \\
\text { intensity }\end{array}$ & $\begin{array}{c}\text { Wave } \\
\text { length }\end{array}$ & $\begin{array}{c}\text { Visibility } \\
\times \\
\text { intensity }\end{array}$ & $\begin{array}{r}\text { Wave } \\
\text { length }\end{array}$ & $\begin{array}{c}\text { Visibility } \\
\times \\
\text { intensity }\end{array}$ \\
\hline $\mathrm{m}_{\mu}$ & & $\mathrm{m}_{\mu}$ & & & \\
400 & $\mathbf{0 . 0 2}$ & 520 & 67.24 & 640 & 15.29 \\
10 & .07 & 30 & 83.89 & 50 & 9.17 \\
20 & .26 & 40 & 94.53 & 60 & 5.14 \\
30 & .80 & 50 & 99.65 & 70 & 2.57 \\
440 & 1.76 & 560 & 99.01 & 680 & 1.34 \\
50 & 3.26 & 70 & 93.73 & 90 & .67 \\
60 & 5.51 & 80 & 84.01 & 700 & .33 \\
70 & 8.70 & 90 & 71.66 & 10 & .16 \\
480 & 13.68 & 600 & 59.14 & 720 & .07 \\
90 & 20.68 & 10 & 46.67 & & $1,028.93$ \\
500 & 32.41 & 20 & 34.90 & & \\
10 & 48.80 & 30 & 23.81 & & \\
\hline
\end{tabular}

The summation of the values of VIR for all wave lengths divided by the summation of $V I$ (which is the value of VIR for magnesium carbonate, for which $R$ is 1 by our choice of units) gives the ratio of the brightness of the color to the brightness of the-magnesium carbonate block.

This process for measuring brightness spectrophotometrically may be summarized in the following three steps:

1. Spectral reflection data for the test sample are obtained by means of a spectrophotometer. These data are recorded as reflection factors relative to those from a magnesium carbonate block for wave lengths, varying by small intervals, such as $10 \mathrm{~m} \mu$, over the entire range of the visible spectrum.

2. Each reflection factor is multiplied by the corresponding VI value taken in Table 5 .

3 . The values of $V I R$, found in (2), for small regular intervals are added together and divided by the sum of the corresponding values of $V I$. (The VIR and the $V I$ curves may be plotted and the areas inclosed by them measured by a planimeter instead of being computed as recommended above.)

\footnotetext{
7 Ibid., second column, Table 2, p. 541. A table of visibility factors slightly different from that given in the report quoted has been since adopted as standard. See B. S. Sc. Paper No. 475, Table 3, last column, page 174; also Trans. Illum. Engrg. Soc., 19, pp. 176 and 614; 1924; and 20, p. 632; 1925.

8 Ibid., first and fourth columns, Table 8, p. 563.
} 
This ratio, VIR to $V I$, is the brightness of the test surface relative to the magnesium carbonate block.

For the simple specification of color (independent of brightness) two systems have been devised-the trichromatic and the monochromatic systems. In the trichromatic system the color is specified as equivalent to a mixture of certain percentages of three hypothetical elementary colors - red, green, and violet-so chosen that their combination in equal amounts would produce white light. In the monochromatic system the color is specified in terms of a homogeneous wave length, which evokes a hue identical with that of the test sample, and a certain purity of color which is the ratio between the brightness of the homogeneous wave length and the brightness of the total sample.

The relation between the two methods of specifying color is shown by the "color triangle," Figure 17.9 The color triangle is laid out with ordinates representing the percentage of red and abscissas representing the percentage of violet, the remainder being green. The specification of color by dominant wave length and purity is represented by the radiating lines and curves centering about the vertex. In this particular chart, to facilitate reading, the straight lines which indicate the hues have been separated from the curves marking the purity values. For example, the color represented by the small circles may be specified either by the trichromatic method as made up of 20 per cent violet, 27 per cent red, and 53 per cent green or by the monochromatic method as having a dominant wave length of $510 \mathrm{~m} \mu$ and a purity of 75 per cent.

The trichromatic method of specification is for our purpose only an auxiliary to the monochromatic; spectrophotometric data are converted first into trichromatic terms and then, by the aid of the color triangle, into terms of dominant wave length and purity.

The hue of an object is dependent upon the character of the illumination, just as we have seen is true in the case of brightness. A surface painted with red lead appears decidedly yellow under a mercury are lamp. The change in color of cloth purchased under artificial light when brought into sunlight is a common experience. As a consequence the quality of the illuminating light has been arbitrarily specified for this purpose of color measurement as average noon sunlight.

It has been determined just how and to what relative extent light of any particular wave length would excite sensations of the red,

o This color triangle is similar to the one used in this work. It is reproduced through the courtesy of Carl W. Keuffel from a chart made by the Keuffel \& Esser Co., plotted from data of Tables 14A and $14 \mathrm{~B}$ in the colorimetry report previously referred to. 


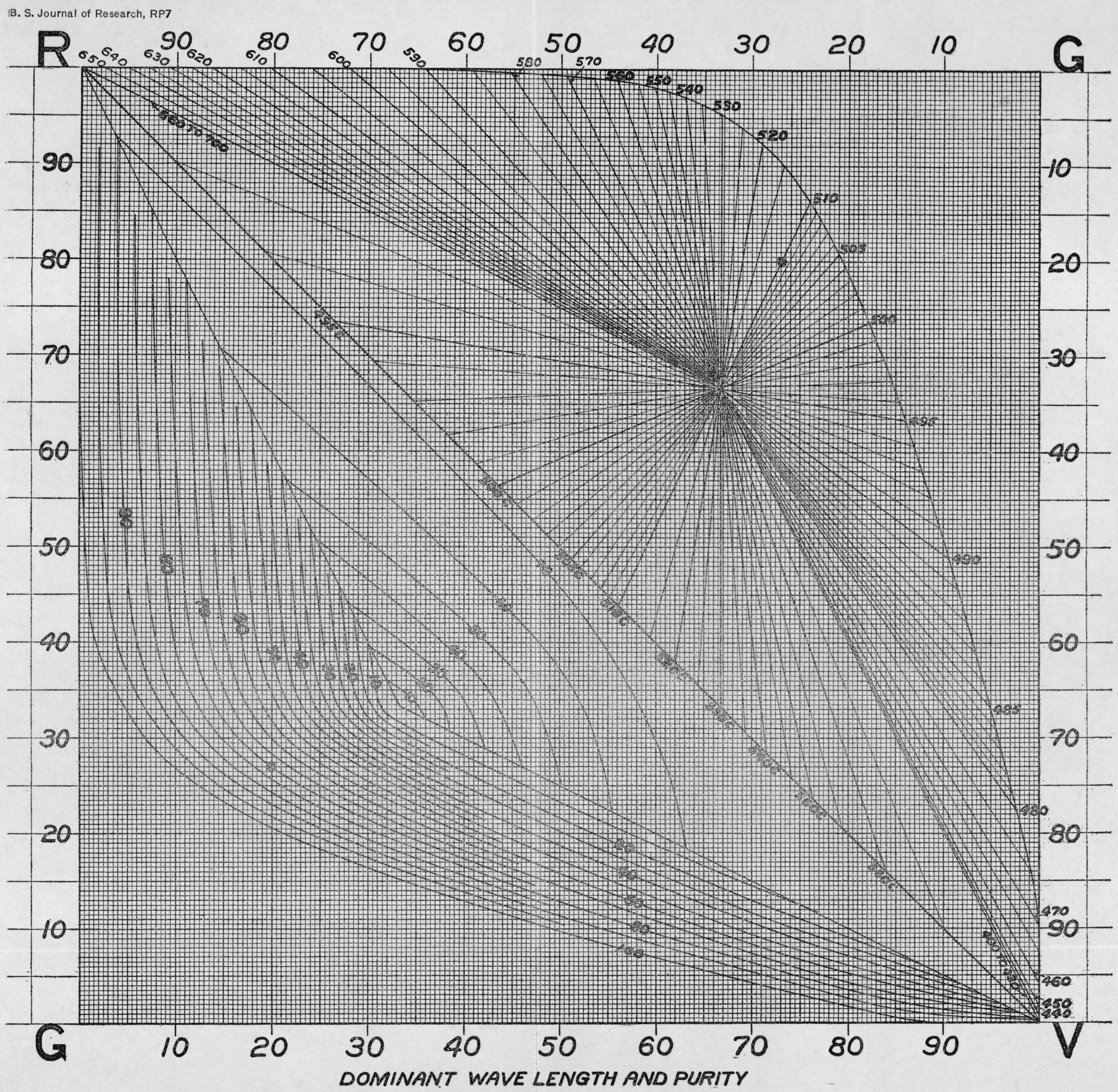

FIG. 17.-Diagrammatic illustration of the relationship between the trichromatic and the monochromatic specifications of color 
green, and violet elementary colors. Thus a certain quantity of energy vibrating as light of $400 \mathrm{~m} \mu$ wave length would excite 253 arbitrary units of the violet, but no red or green, while a ray of 600 $\mathrm{m} \mu$ wave length containing the same amount of energy would excite no violet and much less green than red in the proportion of 196 to 510 .

In view of this, any particular wave length of a reflection spectrum would excite an intensity of elementary color depending upon the intensity of that wave length in the incident light, on the portion of the incident light reflected, and on the excitation values of that wave length; that is, the vibration's ability to excite the three elementary colors. Thus, for any single wave length:

$$
\begin{aligned}
& L_{\mathrm{R}}=R I E_{\mathrm{R}} \\
& L_{\mathrm{G}}=R I E_{\mathrm{G}} \\
& L_{\mathrm{v}}=R I E_{\mathrm{V}}
\end{aligned}
$$

where $L_{\mathrm{R}}, L_{\mathrm{G}}$, and $L_{\mathrm{V}}$ are the amounts of the respective elementary colors that the particular wave length under consideration is equivalent to, where $R$ is the reflection factor, where $I$ is the intensity of the illumination, and where $E_{\mathrm{R}}, E_{\mathrm{G}}$, and $E_{\mathrm{v}}$ are the excitation values for the red, green, and violet elementary colors, respectively.

Summing up the portions of red, of violet, and of green colors over the entire reflection spectrum gives the relative amounts of the three colors which, combined, would produce the color under investigation. These three amounts reduced to a percentage basis are the trichromatic specifications of the test color. By the aid of the relationship represented in Figure 17 these trichromatic percentages can be converted directly in to the monochromatic expression for the test color.

This process of converting the expression for a color from a spectral reflection curve into terms of dominant wave length and purity may be summarized in the following steps 4 to 7 .

4. The mean intensity values for average noon sunlight are multiplied by the corresponding excitation values ${ }^{10}$ for each of the red, green, and violet elementary colors. There result three separate curves, $I E_{\mathrm{R}}, I E_{\mathrm{G}}$, and $I E_{\mathrm{v}}$, the data for which are tabulated in Table 6 .

${ }^{10}$ Ibid. Table 6, p. 549. 
TABLE 6.-Spectral distribution of the excitation intensity product for average noon sunlight

\begin{tabular}{|c|c|c|c|c|c|c|c|}
\hline $\begin{array}{l}\text { Wave } \\
\text { length }\end{array}$ & $I E_{\mathrm{R}}$ & $I E_{\mathrm{G}}$ & $I E_{\mathrm{V}}$ & $\begin{array}{l}\text { Wave } \\
\text { length }\end{array}$ & $I E_{\mathrm{R}}$ & $I E_{\mathrm{G}}$ & $I E_{\mathrm{V}}$ \\
\hline $\begin{array}{r}400 \\
10 \\
20 \\
30 \\
40\end{array}$ & & 567 & $\begin{array}{l}14,168 \\
27,279 \\
42,980 \\
66,795 \\
82,539\end{array}$ & $\begin{array}{r}550 \\
60 \\
70 \\
80 \\
90\end{array}$ & $\begin{array}{l}44,944 \\
48,930 \\
52,520 \\
53,040 \\
53,500\end{array}$ & $\begin{array}{l}64,872 \\
60,690 \\
53,768 \\
42,330 \\
29,600\end{array}$ & $\begin{array}{r}1,908 \\
1,155 \\
728 \\
408\end{array}$ \\
\hline $\begin{array}{r}450 \\
60 \\
70 \\
80 \\
90\end{array}$ & $\begin{array}{l}1,456 \\
4,326\end{array}$ & $\begin{array}{r}1,448 \\
3,686 \\
8,181 \\
12,688 \\
17,830\end{array}$ & $\begin{array}{l}85,975 \\
81,674 \\
70,397 \\
49,192 \\
23,210\end{array}$ & $\begin{array}{r}600 \\
10 \\
20 \\
30 \\
40\end{array}$ & $\begin{array}{l}50,490 \\
45,276 \\
36,375 \\
27,360 \\
18,525\end{array}$ & $\begin{array}{r}19,404 \\
11,074 \\
5,723 \\
2,784 \\
950\end{array}$ & \\
\hline $\begin{array}{r}500 \\
10 \\
20 \\
30 \\
40\end{array}$ & $\begin{array}{r}8,798 \\
16,082 \\
24,698 \\
32,542 \\
39,538\end{array}$ & $\begin{array}{l}27,560 \\
41,642 \\
54,060 \\
60,632 \\
63,918\end{array}$ & $\begin{array}{r}13,038 \\
9,266 \\
6,466 \\
4,558 \\
3,074\end{array}$ & $\begin{array}{l}650 \\
60 \\
70 \\
80 \\
90+\text { +seq. }\end{array}$ & $\begin{array}{r}11,092 \\
6,256 \\
3,620 \\
1,947 \\
2,349\end{array}$ & 282 & \\
\hline
\end{tabular}

5. The reflection factors from the spectral reflection curve from (1) are multiplied by the corresponding ordinates of each of the three curves from (4), Table 6. Three RIE curves result-one for the red, one for the green, and one for the violet.

6. The ordinates of each of the three curves from (5) are summed up. These three sums are then added together. The ratio of each sum to the total of the three is calculated. These ratios are the percentages of red, green, and violet in the reflected light.

7. The ratios from (6) of the red and the violet are plotted in the triangular graph (fig. 17) from which are read directly the purity and the dominant wave length of the sample color under examination.

The writer acknowledges with appreciation the assistance of P. H. Walker, I. G. Priest, and E. R. Weaver in this work.

WAshington, January 8, 1927. 